\title{
Electronic Structure and Bonding Configuration of Histidine on Ge(100)
}

\author{
Hangil Lee, ${ }^{*}$ Young-Sang Youn, ${ }^{\dagger}$ Sena Yang, Soon Jung Jung, ${ }^{\dagger}$ and Sehun Kim ${ }^{\dagger, *}$ \\ Department of Chemistry, Sookmyung Women's University, Seoul 140-742, Korea. *E-mail: easyscan@sookmyung.ac.kr \\ ${ }^{\dagger}$ Molecular-Level Interface Research Center, Department of Chemistry, KAIST, Daejeon 305-701, Korea \\ ${ }^{*}$ E-mail: sehun-kim@kaist.ac.kr \\ Received July 27, 2010, Accepted September 7, 2010
}

\begin{abstract}
The electronic structures and bonding configuration of histidine on $\mathrm{Ge}(100)$ have been investigated with various sample treatments using core-level photoemission spectroscopy (CLPES). Interpretation of the Ge $3 d, \mathrm{C} 1 s, \mathrm{~N} 1 s$, and O $1 s$ core level spectra being included in these systems revealed that both the imino nitrogen in the imidazole ring and the carboxyl group in the glycine moiety concurrently participate in the adsorption of histidine on a $\mathrm{Ge}(100)$ surface at $380 \mathrm{~K}$. Moreover, we could clearly confirm that the imino nitrogen with a free lone pair in the imidazole group adsorbs on $\mathrm{Ge}(100)$ more strongly than the carboxyl group in the glycine moiety by examining systems annealed at various temperatures.
\end{abstract}

Key Words: Histidine, Ge(100), Core-level photoelectron spectroscopy (CLPES), Adsorption, Desorption

\section{Introduction}

In recent decades, efforts to elucidate the adsorption structures of amino acids on metal and semiconductor surfaces have been of great importance not only because of possible industrial applications in technologies such as biosensors and electronic devices, but also due to academic interest stemming from the various functional groups in amino acids. ${ }^{1-5}$ Each amino acid contains a carboxyl group (-COOH), an amino group $\left(-\mathrm{NH}_{2}\right)$, a hydrogen atom $(-\mathrm{H})$, and a side chain (the R-group) attached to the $\alpha$-carbon. Because amino acids contain this variety of functional groups, numerous adsorption structures can arise as a result of intra- or intermolecular competition between the functional groups and the metal or semiconductor surface. ${ }^{6,7}$

Although the adsorption structures of amino acids on metal surfaces have been considered in numerous reports, only a few studies have focused on amino acids adsorbed on semiconductor surfaces. ${ }^{8-13}$ However, results from studies of amino acids on metal surfaces cannot be directly applied to semiconductor systems, since metal and semiconductor surfaces have very different properties. The functionalization of biomolecules is a fascinating field. Elucidating the molecular level behavior of biomolecules at an interface with a solid surface is desirable because such interfacial phenomena lie at the heart of biological

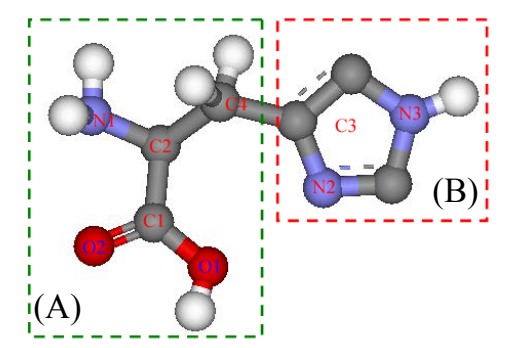

Figure 1. Schematic diagram of the histidine molecule including glycine moiety (A) and imidazole group (B). Each gray, blue, red, or white colored ball indicates carbon $(\mathrm{C})$, nitrogen $(\mathrm{N})$, oxygen $(\mathrm{O})$, or hydrogen $(\mathrm{H})$, respectively. interactions and, hence, have implications for industrial applications in nano-biotechnology and biomedical sciences. ${ }^{1,14,15}$ Therefore, if semiconductor-based bio-applications are to be realized, studies of amino acid adsorption on semiconductor surfaces are needed to determine the fundamental behavior of such systems.

Histidine is an amino acid comprised of an imidazole (IM) ring and a glycine moiety (see Fig. 1). This amino acid has various adsorption structures because the imidazole side chain includes two distinct nitrogen atoms, an imino nitrogen (marked as N2 in Fig. 1) and an amino nitrogen (marked as N3 in Fig. 1), and the glycine moiety has carboxyl and amine groups. ${ }^{11,16,17}$

Recently, Prince et al. used X-ray photoelectron spectroscopy (XPS) to determine the electronic structure of histidine on a $\mathrm{Cu}(110)$ surface as a function of coverage. ${ }^{10}$ At low coverage, they found that the carboxyl group in the glycine part and the imino (N2) nitrogen atom in the imidazole ring were both bound to the $\mathrm{Cu}(110)$ surface. Prompted by these findings, in the present work we used Core-level photoelectron spectroscopy (CLPES) to examine the adsorption structure of histidine on a semiconducting surface $(\mathrm{Ge}(100))$, both to compare the observed behavior with the findings of Prince et al. for histidine on a $\mathrm{Cu}(110)$ surface and to clarify the variation of electronic structure for histidine adsorbed on a $\mathrm{Ge}(100)$ surface as functions of coverage and annealing temperature. To our knowledge, this is the first report on the electronic structure of histidine on $\mathrm{Ge}(100)$.

\section{Experimental Section}

The Ge(100) surface ( $p$-type, $R=0.10-0.39 \Omega$ ) was cleaned by several cycles of sputtering with $1 \mathrm{keV} \mathrm{Ar}^{+}$ions for 20 minute at $700 \mathrm{~K}$, followed by annealing at $900 \mathrm{~K}$ for 10 minute. The cleanness of the $\mathrm{Ge}(100)-2 \times 1$ surface was checked using low energy electron diffraction (LEED). L-Histidine $\left(\mathrm{C}_{6} \mathrm{H}_{9} \mathrm{~N}_{3} \mathrm{O}_{2}\right.$, 99\% purity) was purchased from Aldrich and further purified through several sublimation and pumping cycles to remove all the dissolved gases prior to exposure to the Ge(100) surface. 
To obtain the appropriate vapor pressure for dosing, the dosing line was heated at $320 \mathrm{~K}$ during histidine deposition. Moreover, we confirm its condition using Temperature Programmed desorption (TPD) before we perform experiments of CLPES to clarify deposition of histidine molecules without bond breaking and then we deposited histidine molecules every 30 seconds to confirm a constant deposition rate.

We performed CLPES at the 7B1 beamline of the Pohang Accelerator Laboratory. The Ge $3 d, \mathrm{C} 1 s, \mathrm{~N} 1 s$, and $\mathrm{O} 1 s$ corelevel spectra were obtained with a PHOIBOS 150 electron energy analyzer equipped with a two-dimensional charge-coupled device (2D CCD) detector (Specs $\mathrm{GmbH}$ ) using photon energies of $80,340,460$ and $588 \mathrm{eV}$ to enhance the surface sensitivity. Four binding energies of the core-level spectra were calibrated with respect to that of the clean Au valence band (Fermi energy) and $\mathrm{Au} 4 f$ core level spectrum $(84.0 \mathrm{eV})$ for the same photon energy. The base pressure of the chamber was maintained below 9.5 $10^{-11}$ torr. All of the spectra were recorded in the normal emission mode. The photoemission spectra were carefully analyzed using a standard nonlinear least squares fitting procedure with Voigt functions. ${ }^{18}$

\section{Results and Discussion}

Figure 2(a) shows the Ge $3 d$ core-level spectrum obtained from a clean $\mathrm{Ge}(100)$ surface. The spectrum contains three welldefined features, ${ }^{19,20}$ which can be assigned to bulk Ge atoms $(29.15 \mathrm{eV})$, subsurface Ge atoms $\left(28.95 \mathrm{eV}\right.$, marked as $\left.\mathrm{S}^{\prime}\right)$, and up-atoms of asymmetric Ge dimers $(28.62 \mathrm{eV}$, marked as $\mathrm{S})$. After confirming that the $\mathrm{Ge}(100)$ surface was clean, we exposed it to histidine at $380 \mathrm{~K}$ to make good progress of the adsorption of histidine molecules on the surface. Fig. 2(b) shows the Ge $3 d$ core level spectrum obtained after exposure of the surface to $0.20 \mathrm{ML}$ of histidine. The Ge $3 d$ core level spectra of

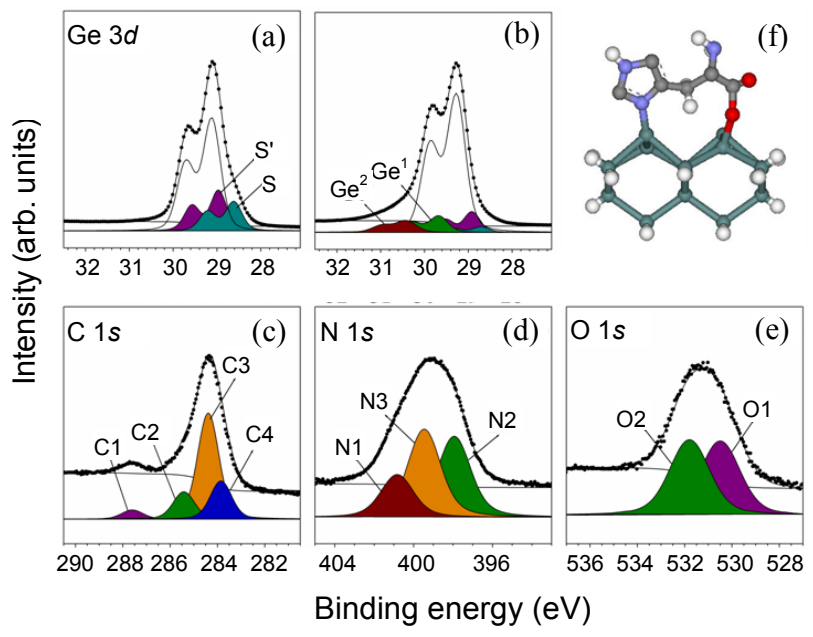

Figure 2. Ge $3 d$ core-level spectra for histidine adsorbed on $\mathrm{Ge}(100)$. (a) clean Ge $3 d$ core level spectrum, (b) Ge $3 d$, (c) C $1 s$, (d) $\mathrm{N} 1 s$, and (e) $\mathrm{O} 1 s$ core level spectra obtained from 0.20 ML of histidine adsorbed on $\mathrm{Ge}(100)$ at $380 \mathrm{~K}$. The dots are experimental values and the solid lines represent the results of peak fitting. (f) Schematic adsorbed structure for histidine on $\mathrm{Ge}(100)$. Each gray, white, blue, red, dark cyan colored ball indicates carbon $(\mathrm{C})$, hydrogen $(\mathrm{H})$, nitrogen $(\mathrm{N})$, oxygen $(\mathrm{O})$, and $\mathrm{Ge}(100)-2 \times 1$ surface, respectively.
$\mathrm{Ge}(100)$ after exposure to histidine is remarkably different from the spectra before exposure. The adsorption of histidine molecules induces a decrement in the intensity of the surface peak (S) and subsurface peak ( $\left.\mathrm{S}^{\prime}\right)$, indicating modification of the electron densities of the Ge atoms at the surface. In addition, histidine adsorption caused the emergence of two new peaks (marked as $\mathrm{Ge}^{1}: 29.61 \mathrm{eV}$ and $\mathrm{Ge}^{2}: 30.30 \mathrm{eV}$ ), which are located higher than the bulk Ge $3 d$ peak $(29.15 \mathrm{eV})$. Considering the Pauling electronegativity for the nitrogen atom $(\mathrm{PE}=$ $3.1)$ and oxygen atom $(\mathrm{PE}=3.6)$, we assigned the peaks to a Ge-N (N2 or N3) bonding feature $\left(\mathrm{Ge}^{1}: 29.61 \mathrm{eV}\right)$ and a Gecarboxyl bonding feature $\left(\mathrm{Ge}^{2}: 30.30 \mathrm{eV}\right)$, respectively. It is possible that the glycine moiety binds to the substrate via the nitrogen of its amine moiety (marked as N1 in Fig. 1) rather than the carboxyl oxygen (marked as $\mathrm{O} 1$ in Fig. 1). However, if this were the case, we would not see the higher binding energy peak $(30.30 \mathrm{eV})$ in the $\mathrm{Ge} 3 d$ core-level spectrum. Thus, we can exclude the existence of the Ge-amine bonding feature. Our findings thus indicate that the histidine molecule is concurrently adsorbed on $\mathrm{Ge}(100)$ with two bonding configurations involving glycine moiety (carboxyl group) and the imidazole ring.

We also examined three core level spectra (C $1 s, \mathrm{~N} 1 s$, and $\mathrm{O} 1 s$ ) being included in the histidine molecule to track the electronic structure for histidine on Ge(100) surface. Fig. 2(c) shows the $\mathrm{C} 1 \mathrm{~s}$ core level spectrum obtained after exposure of $0.20 \mathrm{ML}$ histidine at $380 \mathrm{~K}$. The spectrum exhibits four distinct bonding features, thereby confirming that histidine is well adsorbed on the $\mathrm{Ge}(100)$ surface without bond breaking. Considering electronegativity and energy resolution, we assigned the bonding features, designated as $\mathrm{C} 1, \mathrm{C} 2, \mathrm{C} 3$, and $\mathrm{C} 4$, to COO- $(287.5 \mathrm{eV}$ : $\mathrm{C} 1), \mathrm{C}-\mathrm{NH}_{2}\left(285.5 \mathrm{eV}\right.$ : C2), imidazole (284.5 eV: C3), and $\mathrm{CH}_{2}-$ (284.0 eV: C4), respectively.

On the basis of the analysis of Ge $3 d$ and $\mathrm{C} 1 s$ core level spectra presented above, we tentatively concluded that histidine molecules adsorbed on the Ge(100) surface via the imidazole group and the carboxyl group being included in the glycine moiety. However, we could not determine which nitrogen (N2 or N3) in the imidazole group participated in the binding to the $\mathrm{Ge}(100)$ surface from these information. We should, however, be able to elucidate the bonding configuration of the imidazole group by examining the N $1 s$ core level spectra. Hence, we also obtained N $1 s$ core level spectrum for $0.20 \mathrm{ML}$ (Fig. 2(d)) of histidine adsorbed on $\mathrm{Ge}(100)$ at $380 \mathrm{~K}$. It clearly shows three distinct $\mathrm{N}$ peaks corresponding to the three $\mathrm{N}$ atoms being included in the histidine molecule, indicating that the adsorbed histidine molecules remained intact under these conditions. Considering electronegativity and bonding configurations, we could clearly assign the $\mathrm{N} 1(400.9 \mathrm{eV}), \mathrm{N} 2(398.2 \mathrm{eV})$, and N3 $(399.4 \mathrm{eV})$ features to the amine group in the glycine moiety, the imino nitrogen and the amino nitrogen in the imidazole ring, respectively.

Recently, Prince et al. ${ }^{10}$ reported the electronic structure of $0.40 \mathrm{ML}$ histidine on a $\mathrm{Cu}(110)$ surface at $300 \mathrm{~K}$. Similar to our results, they assigned the binding energies of N1, N2, and N3 as $400.4,398.6$, and $399.6 \mathrm{eV}$, respectively. Therefore, our findings demonstrate that histidine molecules adsorb on the $\mathrm{Ge}(100)$ surface without bond breaking although we obtained it at $380 \mathrm{~K}$. 
Having confirmed that histidine can be adsorbed on $\mathrm{Ge}(100)$ via two bonding groups (imidazole and glycine), we now focus on elucidating in detail the variations in adsorption and electronic structures using the same method as was used above to obtain the C $1 s$ core level spectra shown in Fig. 2(c).

Finally, we concurrently obtained $\mathrm{O} 1 s$ core level spectra for $0.20 \mathrm{ML}$ (Fig. 2(e)) of histidine adsorbed on $\mathrm{Ge}(100)$ maintained at $380 \mathrm{~K}$. By considering electronegativity, we can clearly divide the spectral features into two bonding states: feature $\mathrm{O} 1$ $(530.4 \mathrm{eV})$ induced by $\mathrm{Ge}-\mathrm{O}-$; and feature $\mathrm{O} 2(531.8 \mathrm{eV})$ originating from $-\mathrm{C}=\mathrm{O}$. As a result, we can demonstrate that nitrogen being included in the imidazole ring and the carboxyl group in the glycine moiety concurrently participate in the adsorption of histidine on a $\mathrm{Ge}(100)$ surface at $380 \mathrm{~K}$.

Having confirmed that the histidine molecules adsorbed on the $\mathrm{Ge}(100)$ surface without bond breaking under our experimental conditions, we next focused on tracking the stable adsorption structure of histidine as a function of annealing temperature. From the results acquired from Fig. 2, we can't clearly demonstrate which nitrogen being included in imidazole group can be participated in adsorption to $\mathrm{Ge}(100)$ surface. To understand precisely the bonding configuration of adsorbed histidine on $\mathrm{Ge}(100)$, we also performed CLPES measurements as a function of annealing temperature. Hence, we concurrently recorded a series of temperature dependent $\mathrm{C} 1 s, \mathrm{~N} 1 s$, and $\mathrm{O}$ $1 s$ core-level spectra for adsorbed histidine on a $\mathrm{Ge}(100)$ surface as we increased the annealing temperature from $420 \mathrm{~K}$ to $570 \mathrm{~K}$ for 5 minute after exposing the substrate to $0.20 \mathrm{ML}$ of histidine at $380 \mathrm{~K}$. Through the spectral analysis of the temperature-dependent change in bonding character, we expect that we can obtain the information on the desorption process. Hence, the observation of annealing effects is very important and useful as it sheds light on the desorption process and the stable and exact adsorption structure of histidine on $\mathrm{Ge}(100)$.

Figure 3(a) shows the $\mathrm{C} 1 s$ core level spectrum obtained after annealing at $420 \mathrm{~K}$ for 5 minutes. Comparison of this spectrum with that obtained before annealing (Fig. 2(d)) discloses two

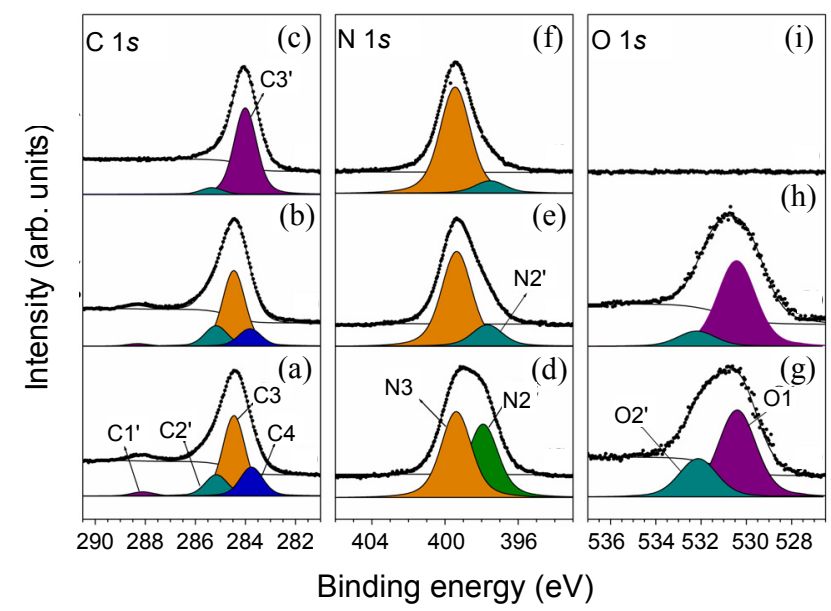

Figure 3. $\mathrm{C} 1 s, \mathrm{~N} 1 s$, and $\mathrm{O} 1 s$ core-level spectra for histidine adsorbed on $\mathrm{Ge}(100)$. (a), (d), and (g) $0.20 \mathrm{ML}$ of histidine annealed for $5 \mathrm{~min}-$ ute at $420 \mathrm{~K} \pm 10 \mathrm{~K},(\mathrm{~b}),(\mathrm{e})$, and (h) annealed at $500 \mathrm{~K} \pm 10 \mathrm{~K}$, (c), (f), and (i) annealed at $570 \mathrm{~K} \pm 10 \mathrm{~K}$. The dots are experimental values and the solid lines represent the results of peak fitting. interesting results, namely changes in the COO- feature $(\mathrm{C} 1)$ and $\mathrm{C}-\mathrm{NH}_{2}$ feature $(\mathrm{C} 2)$. Specifically, the $\mathrm{C} 1$ peak shifts toward higher binding energy, from $287.5 \mathrm{eV}$ to $288.2 \mathrm{eV}$ (marked as $\mathrm{C} 1$ '), and the $\mathrm{C} 2$ peak moves to lower binding energy, from $285.5 \mathrm{eV}$ to $285.3 \mathrm{eV}$ (marked as $\mathrm{C} 2$ '). To account for these changes in electronic structure, we provide two hypotheses. First, the bonding between the carboxyl group and the $\mathrm{Ge}(100)$ surface becomes weak and the histidine starts to be desorbed or to recombine. In other words, the binding energy shift toward higher binding energy indicates that the Ge-COO bond is weakened by annealing. Second, the amine group attached to the $\mathrm{C} 2$ carbon also undergoes desorption or recombines. In other words, the bonding strength between $\mathrm{C} 2$ and $\mathrm{C} 4$ becomes strong due to desorption of the amine group. On the basis of these findings, we can speculate that a new double bond character $(\mathrm{C}=\mathrm{C}-)$ or a $-\mathrm{CH}_{2}$ bonding feature is present instead of the amine group. To explore these two possibilities, we consider the $\mathrm{N} 1 s$ and $\mathrm{O}$ $1 s$ core level spectra. In addition, we acquired the $\mathrm{C} 1 s$ core level spectrum obtained after annealing at $500 \mathrm{~K}$ for 5 minutes (Fig. 3(b)). This spectrum does not show a remarkable change of the electronic structure compared with that obtained after annealing at $420 \mathrm{~K}$ (Fig. 3(a)), although the intensity of the features decreases, indicating progression of the desorption process. After annealing at $570 \mathrm{~K}$ for 5 minutes (Fig. 3(c)), however, a huge change in the electronic structure occurs: the $\mathrm{C} 1$ (carboxyl group) and $\mathrm{C} 4\left(-\mathrm{CH}_{2}\right)$ features have disappeared and the intensity of the $\mathrm{C} 2$ ' peak is remarkably decreased. Moreover, a new peak (marked as C3') emerged at $284.1 \mathrm{eV}$ in place of the $\mathrm{C} 3$ peak. From this result, we can speculate that the carboxyl group is fully broken and desorbed. Also, the imidazole ring is broken and a different bonding feature has emerged. These findings thus indicate that, at an annealing temperature of $570 \mathrm{~K}$, histidine molecules adsorbed on a Ge(100) surface are fully broken, and only fragments remain on the surface.

By a similar process, we also obtained the $\mathrm{N} 1 s$ core level spectra at various annealing temperatures. Fig. 3(d) shows the $\mathrm{N} 1 s$ core level spectrum recorded after annealing at $420 \mathrm{~K}$ for 5 minutes. Comparison of this spectrum with that of the system before annealing reveals a large change in the electronic structure, in that the feature arising from the amine group in the glycine moiety (marked as N1) completely disappears after annealing. This finding suggests that the binding energy shift of the $\mathrm{C} 2$ feature may originate from bond breaking of the $\mathrm{C}-\mathrm{NH}_{2}$ feature. From the disappearance of amine group being included in glycine moiety, we can infer that our speculation is correct as we consider in Fig. 2. On the other hand, we observed that the other nitrogen features being included in the imidazole ring (marked as N2 and N3) still remained unchanged at this annealing temperature. Hence, the spectral data indicate that at this annealing temperature, the imidazole group is not broken and that it is adsorbed on $\mathrm{Ge}(100)$ via strong bonds. Moreover, our observation that the amine group included in the glycine moiety disappears establishes that the two bonding features adsorbed on the $\mathrm{Ge}(100)$ surface at this annealing temperature (420 K) are the oxygen included in the carboxyl group of the glycine moiety and the nitrogen included in the imino group of the imidazole ring.

We then continuously increased the annealing temperature 
Table 1. Changes in binding energy for three core level spectra of histidine on a Ge(100) surface as a function of coverage and annealing temperature.

\begin{tabular}{|c|c|c|c|}
\hline \multirow{2}{*}{ Annealing Temperature $\pm 10(\mathrm{~K})$} & $\mathrm{C} 1 s(\mathrm{eV})$ & $\mathrm{N} 1 s(\mathrm{eV})$ & $\mathrm{O} 1 s(\mathrm{eV})$ \\
\hline & $\mathrm{C} 1\left(\mathrm{C} 1^{\prime}\right) / \mathrm{C} 2\left(\mathrm{C} 2^{\prime}\right) / \mathrm{C} 3\left(\mathrm{C} 3^{\prime}\right) / \mathrm{C} 4$ & N1/N3/N2(N2') & $\mathrm{O} 1 / \mathrm{O} 2\left(\mathrm{O} 2{ }^{\prime}\right)$ \\
\hline $0.20 \mathrm{ML}$ at $380 \mathrm{~K}$ & $287.5 / 285.5 / 284.5 / 284.0$ & 400.9/399.4/398.2 & $530.4 / 531.8$ \\
\hline Annealed at $420 \mathrm{~K}$ & $(288.2) /(285.3) / 284.5 / 284.0$ & $(\mathrm{n} / \mathrm{a}) / 399.4 / 398.2$ & $530.4 /(532.2)$ \\
\hline Annealed at $500 \mathrm{~K}$ & $(288.2) /(285.3) / 284.5 / 284.0$ & $(\mathrm{n} / \mathrm{a}) / 399.4 /(397.6)$ & $530.4 /(532.2)$ \\
\hline Annealed at $570 \mathrm{~K}$ & $(\mathrm{n} / \mathrm{a}) /(285.3) /(284.1) /(\mathrm{n} / \mathrm{a})$ & $(\mathrm{n} / \mathrm{a}) / 399.4 /(397.6)$ & $\mathrm{n} / \mathrm{a}$ \\
\hline
\end{tabular}

and tracked the change in the electronic structure to determine which nitrogen (N2 or N3) is adsorbed on the Ge(100) surface. Fig. 3(e) shows the N $1 s$ core-level spectrum recorded after annealing at $500 \mathrm{~K}$ for 5 minutes. Compared to the $420 \mathrm{~K}$ spectrum, the intensity of the $\mathrm{N} 2$ feature is remarkably decreased at $500 \mathrm{~K}$. Moreover, the binding energy shifts from $398.2 \mathrm{eV}$ (marked as N2) to $397.6 \mathrm{eV}$ (marked as N2'), indicating a change in the electronic structure in the imidazole ring. From this result, we can speculate that the imidazole ring starts to be desorbed at this annealing temperature. In contrast to the imino nitrogen, the feature corresponding to the amino nitrogen (marked as N3) does not change on going from $420 \mathrm{~K}$ to $500 \mathrm{~K}$, indicating that imidazole ring is not broken at this annealing temperature. Finally, we increased the annealing temperature to $570 \mathrm{~K}$ for 5 minute. As shown in Fig. 3(f), the intensity of the N2' feature is remarkably decreased whereas those of the N3 bonding feature remain. This indicates that the N2' bonding feature is greatly diminished or rearranged on the surface, although the binding energy of both peaks remains unchanged. The spectral data thus show that the imino nitrogen (N2) is one of the bonding sites with $\mathrm{Ge}(100)$. From the analysis of the $\mathrm{C} 1 s$ and $\mathrm{N} 1 s$ core level spectra, we could obtain the important information regarding the exact adsorption sites.

Finally, we concurrently obtained O $1 s$ core level spectra with varying the annealing temperature. Fig. $3(\mathrm{~g})$ shows the $\mathrm{O} 1 \mathrm{~s}$ core level spectrum recorded after annealing at $420 \mathrm{~K}$ for 5 minutes. Compared to the system before annealing, the intensity of the $\mathrm{O} 2$ feature is remarkably decreased whereas that of the $\mathrm{O} 1$ bonding feature remains unchanged. These findings indicate that the $-\mathrm{C}=\mathrm{O}$ bonding feature (marked as $\mathrm{O} 2^{\prime}$ ) included in the glycine moiety starts to be desorbed and, as a result, this peak shifts toward higher binding energy $(532.2 \mathrm{eV})$. On increasing the annealing temperature to $500 \mathrm{~K}$ for 5 minutes (Fig. 3(h)), the intensity of the $\mathrm{O} 2$ ' bonding feature decreased markedly compared to the $420 \mathrm{~K}$ spectrum whereas that of $\mathrm{O} 1$ remained unchanged, indicating an acceleration of the $-\mathrm{C}=\mathrm{O}$ desorption process. Finally, when the annealing temperature was increased to $570 \mathrm{~K}$ for 5 minute (Fig. 3(i)), no oxygen peaks were found, indicating complete desorption of the carboxyl group from the $\mathrm{Ge}(100)$ surface. The changes in binding energies for histidine molecules adsorbed on a Ge(100) surface under various sample treatments are summarized in Table 1.

\section{Conclusions}

We have investigated the adsorption and desorption structures of the bonding states of histidine adsorbed on a $\mathrm{Ge}(100)$ surface using CLPES. Our results confirm that, at $380 \mathrm{~K}$, both the imino nitrogen (N2) in the imidazole ring and the carboxyl group (O1) of the glycine moiety concurrently participate in the adsorption of histidine on a $\mathrm{Ge}(100)$ surface. In addition, through an analysis of the temperature-dependent spectra of histidine adsorbed on a Ge(100) surface using CLPES, we could corroborate that the imidazole group is adsorbed on $\mathrm{Ge}(100)$ stronger than glycine moiety and histidine is completely desorbed from $\mathrm{Ge}(100)$ at $570 \mathrm{~K}$.

Acknowledgments. We thank Dr. Chan-Cuk Hwang and Han$\mathrm{Na}$ Hwang for supporting the CLPES experiments at the 7B1 beamline in the Pohang Accelerator Laboratory. This research was supported by National Research Foundation of Korea Grant funded by the Korean Government (KRF-2008-314-C00169). This research was also supported by Basic Science Research Program through the National Research Foundation of Korea (NRF) funded by the Ministry of Education, Science and Technology (2010-0001950).

\section{References}

1. Filler, M. A.; Bent, S. F. Prog. Surf. Sci. 2003, 73, 1.

2. Whaley, S. R.; English, D. S.; Hu, E. L.; Barbara, P. F.; Belcher, A. M. Nature 2000, 405, 665-668.

3. Goede, K.; Busch, P.; Grundmann, M. Nano. Lett. 2004, 4, 2115 2120.

4. Loscutoff, P. W.; Bent, S. F. Annu. Rev. Phys. Chem. 2006, 57, 467-495.

5. Ardalan, P.; Davani, N.; Musgrave, C. B. J. Phys. Chem. C 2007, 111, 3692-3699.

6. Wolkow, R. A. Annu. Rev. Phys. Chem. 1999, 50, 413.

7. Hamers, R. J.; Coulter, S. K.; Ellison, M. D.; Hovis, J. S.; Padowitz, D. F.; Schwartz, M. P.; Greenlief, C. M.; Russell, J. N. Acc. Chem. Res. 2000, 33, 617 .

8. Gao, F.; Li, Z.; Wang, Y.; Burkholder, L.; Tysoe, W. T. J. Phys. Chem. C 2007, 111, 9981-9991.

9. Zhao, X.; Yan, H.; Zhao, R. G.; Yang, W. S. Langmuir 2003, 19, 809-813.

10. Feyer, V.; Plekan, O.; Skála, T.; Cháb, V.; Matolín, V.; Prince, K. C. J. Phys. Chem. B 2008, 112, 13655-13660.

11. Youn, Y.-S.; Jung, S.-J.; Lee, H.; Kim, S. Langmuir 2009, 25, 7438.

12. Lee, H.; Youn, Y.-S.; Kim, S. Langmuir 2009, 25, 12574

13. Youn, Y.-S.; Lee, H.; Kim, S. Chem. Phys. Chem. 2010, 11, 354

14. Smith, R. K.; Lewis, P. A.; Weiss, P. S. Prog. Surf. Sci. 2004, 75, 1.

15. Sarikaya, M.; Tamerler, C.; Jen, A. K. Y.; Schulten, K.; Baneyx, F. Nat. Mater. 2003, 2, 577.

16. Zubavichus, Y.; Zharnikov, M.; Yang, Y.; Fuchs, O.; Heske, C.; Umbach, E.; Tzvetkov, G.; Netzer, F. P.; Grunze, M. J. Phys. Chem. B 2005, 109, 884.

17. Xue, G.; Dong, J.; Sun, Y. Langmuir 1994, 10, 1477.

18. Schreier, F. J. Quant. Spectros. Radiat. Transfer. 1992, 48, 743-762.

19. Landmark, E.; Karlsson, C. J.; Johansson, L. S. O.; Uhrberg, R. I. G. Phys. Rev. B 1994, 49, 16523-16533.

20. Jung, S. J.; Youn, Y.-S.; Lee, H.; Kim, K.-J.; Kim, B.; Kim, S. J. Am. Chem. Soc. 2008, 130, 3288-3289. 